\title{
A Novel Spectrally Efficient Asynchronous Multi-Channel MAC Using a Half-Duplex Transceiver for Wireless Networks
}

\author{
Abdullah Devendiran (1), Tarek Sheltami * (1) and Ashraf Mahmoud \\ Department of Computer Engineering, King Fahd University of Petroleum and Minerals, Dhahran 31261, \\ Saudi Arabia; abdullahd@kfupm.edu.sa (A.D.); ashraf@kfupm.edu.sa (A.M.) \\ * Correspondence: tarek@kfupm.edu.sa; Tel.: +966-13-860-4678
}

Received: 8 December 2017; Accepted: 29 January 2018; Published: 30 January 2018

\begin{abstract}
Multi-channel medium access control (MAC) protocols maximize network performance by enabling concurrent wireless transmissions over non-interfering channels. Despite physical layer advancements, the underlying IEEE 802.11 MAC standard cannot fully exploit features and support high-performance applications. In this work, we propose the novel spectrally efficient asynchronous multi-channel MAC (SA-MMAC) protocol for wireless networks using a single half-duplex transceiver. A full-duplex mode of operation on data channels reduces the signaling overhead and boosts the spectrum efficiency. A revamped contention mechanism of IEEE 802.11 addresses the multi-channel hidden terminal problem, and a jamming signal from the receiver addresses the collisions in control signals. Furthermore, the control channel is used for data transmissions to increase the bandwidth utilization but under a restricted half-duplex mode to avoid causing a bottleneck situation. The simulator is tested for correctness. The results suggest that the protocol can work well on 3, 4, or 12 concurrent channels with high node density, providing about 12.5 times more throughput than IEEE 802.11 and $18 \%$ to $95 \%$ more throughput than its multi-channel variants under saturated traffic conditions.
\end{abstract}

Keywords: multi-channel MAC; 802.11 wireless networks; asynchronous; control channel reuse; half-duplex transceiver; event-driven simulation; python simulator; multi-channel hidden terminal

\section{Introduction}

The medium access control (MAC) layer significantly impacts network throughput, as it co-ordinates the channel access between wireless nodes. Despite physical layer advancements, the underlying IEEE 802.11 protocol cannot fully exploit features and support high-performance applications. The IEEE standard [1] defines multiple channels for communication at the physical layer. IEEE standard $802.11 \mathrm{~b}$,g specifies three orthogonal or non-overlapping channels which are $22 \mathrm{MHz}$ wide, and IEEE 802.11a specifies 12 non-overlapping channels: 4 channels of $20 \mathrm{MHz}$ each in the upper, lower, and middle U-NII bands. When orthogonal channels are used, concurrent transmissions can co-exist on multiple channels without causing interference to each other. A transceiver can switch easily between various channels, but IEEE 802.11 is designed only for single-channel operation. With the dense deployment of wireless nodes becoming a reality in the near future, the study of multi-channel protocols to maximize utilization of spectrum resources is an issue of peculiar interest.

A deterrent issue in multi-channel protocols affecting performance is the multi-channel hidden terminal problem wherein the nodes performing data transmission on data channels miss the control packets exchanged on the control channel. Due to missing information on channel status, the pair of nodes may inadvertently choose a busy channel and start data exchange, causing a collision. Of the existing multi-channel MAC protocols, dedicated control channel-based [2,3] and 
time synchronization-based protocols $[4,5]$ handle this issue effectively. The dedicated control channel protocols eliminate the multi-channel hidden terminal issue by having two transceivers, one for control signaling, and other for data transfer, and the nodes are aware of the neighbor activities all the time. However, having two transceivers per node increases the cost, energy consumption, and size. In a dense deployment of nodes with no existing power infrastructures, the usage of nodes with expensive hardware and high power consumption is not feasible. Also, dedicating an entire channel for control exchanges decreases the bandwidth available for data transmissions. Moreover, as the density of nodes increases and a large number of data channels are present, the control channel becomes a serious bottleneck. This makes the dedicated control channel protocols with multiple transceivers less attractive. As for time synchronization-based protocols, the time is divided into control and data phases. The contention for channels occurs during the control phase and the nodes that succeed in negotiations are involved in data transfer during the data phase. All nodes listen to each other during the control phase and are aware of the control exchanges; the multi-channel hidden terminal problem is removed. However global synchronization is a difficult task as it involves considerable overhead and complexity. Distributed or asynchronous multi-channel protocols using single antennas are highly susceptible to multi-channel hidden terminal issues. The authors of [6] proposed a back-off mechanism to overcome this by mandating a waiting time for nodes in successful data transfer before the next contention. Our protocol does include a waiting period but does not mandate a complete data transmission time. In [7], the authors suggest that a careful channel selection strategy can avoid the hidden terminal problem to a reasonable extent.

Another issue in multi-channel protocols is that the loss of control exchanges due to collisions in request-to-send (RTS) and clear-to-send (CTS); i.e., RTS-RTS or RTS-CTS collisions, can lead to information loss. Due to loss of control exchanges, a node may select a data channel in use and cause a data collision. The authors of [8] suggest that if a data channel in use is selected inadvertently, the neighbors can issue an invalid signal and stop the on-going negotiation. Avoiding collisions in multi-channel hidden terminals and control handshakes greatly enhances the network throughput. A strategy in multi-channel protocols to increase the throughput is to employ a batch-mode operation wherein multiple data frames are allowed to transmit per handshake [9] reducing the contention and amortizing the handshake overhead. Keeping the multiple frames allowed per handshake to an optimum number is mandatory in this approach, otherwise other nodes will suffer a high degree of starvation before getting a channel to transmit.

In this paper, a distributed multi-channel MAC protocol using a single transceiver is proposed, which is named the spectrally efficient asynchronous multi-channel (SA-MMAC) protocol. In the proposed protocol, a receiver node, on receiving data successfully, sends data to the sender as a piggy-backed acknowledgment, skipping the control exchanges and reducing the signaling overhead. A full-duplex MAC design was investigated in [10,11]. However, the design in [10] requires an array of physical layer modifications and that in [11] employs a dedicated control channel. The main contributions of this paper can be summarized as follows:

- We propose a novel, simple-to-implement multi-channel MAC protocol to coordinate multiple channel access using a single half-duplex transceiver without the need for time synchronization.

- The protocol extends the IEEE 802.11 single-channel back off/contention mechanism and load balances the parallel transmissions to multi-channels.

- The protocol increases the spectral efficiency and reduces the signaling overhead by allowing a full-duplex mode of operation in data channels.

- The protocol promotes spatial channel re-use by using a control channel for data transmissions and prevents control channel bottlenecks by strict enforcement of half-duplex mode in the control channel.

- The deterrent multi-channel hidden terminal problem is addressed by a revamped contention mechanism wherein the success node pair waits additional time in the maximum data frame before starting to contend. 
- The control signals collisions (RTS-RTS, RTS-CTS) are handled by a jamming signal from receiver after sending the CTS.

The rest of the paper is organized as follows. The operational details of the SA-MMAC protocol are described in Section 2. In Section 3, the details of the simulator validation are provided. A performance evaluation is presented in Section 4 to demonstrate the effectiveness of our proposed protocol. Finally, some conclusions are drawn in Section 5.

\section{The Spectrally Efficient Asynchronous Multi-Channel MAC (SA-MMAC) Protocol}

\subsection{System Model}

In the design of the proposed SA-MMAC protocol, there are two important features:

- A distributed network of $\mathrm{N}$ nodes, with each node equipped with a half-duplex transceiver. A node can transmit or receive at any given time but not both. A node transmitting on a data channel cannot listen to control signals on the control channel.

- K orthogonal channels of equal bandwidth. Simultaneous transmissions can happen on these channels without interfering with each other. One channel is configured for control exchanges and other K-1 channels are configured for data transfer. The control channel is reused for data transfer when possible.

The proposed protocol is a logical extension of IEEE 802.11 and is assumed to have IEEE 802.11-like behavior in other layers of the TCP/IP stack. The channel conditions are assumed to be ideal and error-free and the packets are lost only due to collisions. For the protocol design, we modify the IEEE 802.11 control packets to add some fields. The control frames in SA-MMAC are:

- $\quad$ RTS (request-to-send): is sent when a sender has data to send. The RTS is modified to contain additional information such as the available channel list (ACL) and data transfer time.

- CTS (clear-to-send): is sent to acknowledge the incoming RTS. The frame is modified to contain the selected channel and data transfer time.

- RES (reservation): is sent to confirm neighbors of the on-going transmission. The frame is similar to that of the CTS.

\subsection{Operations of the SA-MMAC Protocol}

The basic idea of our proposed SA-MMAC protocol consists of four aspects.

(1) Full-duplex mode of operation: the receiver node can operate in a full-duplex mode and skip the control overhead signaling only one successful control handshake.

(2) Re-use of the control channel: the control channel is used for data transmissions but is restricted to half-duplex mode operation so as to obtain balance between reuse of the channel and prevention of a bottle-neck at the control channel.

(3) Contention mechanism: multi-channel problems, such as the hidden terminal, are controlled by additional waiting time for the success node pair after data transmission.

(4) Collision avoidance. Finally, the collisions in control signals (RTS/CTS) are handled by an additional jamming signal from the receiver after sending a CTS.

Spectrally efficient asynchronous multi-channel MAC (SA-MMAC) uses a single transceiver and listens to a commonly assigned control channel by default. When a node is ready to send data, it first issues a request-to-send (RTS) on the control channel which includes a list of available channels on the sender side. The receiver, on getting the RTS, checks its list of free channels and selects a commonly available channel. If no common channel is available, or if the node is busy in data exchange, the RTS request is ignored; otherwise a clear-to-send (CTS) is issued. The receiver then switches to the data channel and gets ready to receive data. The sender, on receiving the CTS, announces the selected data channel and channel release time to its neighbors in a frame called reservation (RES). Then, it switches 
to the data channel and starts sending data. Once the data transfer is complete, the receiver sends an acknowledgement (ACK). The neighboring nodes which hear the control exchanges freeze their back-off timers and defer from contention for the duration of transmission. Nodes will send the RTS, CTS, and RES on the control channel and switch to the agreed data channel for data transfer. When the process is complete, the sender-receiver switch to the control channel and take cognizance of the control messages exchanged. The contention and back-off mechanism on the control channel follow IEEE 802.11.

The working of the SA-MMAC protocol is illustrated in Figure 1. Node A has data to send to B and issues a RTS on the control channel $(\mathrm{CCH})$ with the list of available channels. The neighbors of $\mathrm{A}$ defer until after the RTS frame + Data Inter-Frame Space (DIFS) time. B, on receiving the RTS, chooses a common channel. Since B has data to send to A, B adds its own data transfer time to the data transfer time of A indicated in the RTS and sends the CTS to A. The neighbors of B defer from contention until the data transfer time, as indicated in the CTS signal. After the Short Inter-Frame Space (SIFS) time, the receiver sends the RES signal again. Nodes which missed the CTS signal due to collisions can hear the RES and become cognizant of the data transfer. Node A, on receiving the CTS, sends the RES to its neighbors. Then, nodes A,B switch to the selected data channel and transfer data. B, on receiving DATA from $A$, sends back its DATA as a piggy-backed acknowledgement. A on receiving DATA (B) knows that B received its DATA (A) successfully. It then sends acknowledgment (ACK) for DATA (B). The nodes then switch to the control channel and wait for the mandatory time of a maximum data frame and start contending again as per the IEEE 802.11 contention mechanism. When the data transfer of A-B is going on, nodes $\mathrm{C}$ and $\mathrm{D}$ successfully negotiate and start the data transfer process on channel 2 . However, in this case since $D$ does not have any data to send to $C$, it simply sends an acknowledgment after receiving DATA from C. In the scenario described in Figure 1, let us assume the data channels are 2. When $\mathrm{E}$ wants to send data to $\mathrm{F}$, it sees all the data channels are occupied. Hence, E selects the control channel (CCH) for data transfer and sends the RTS. F also has data to send to $\mathrm{E}$, but it skips its data frame, as control channel is used for data transfer. Hence, the CTS from F just includes the data transfer time for E. Our protocol uses a synchronous mode of operation and has some important features which solve commonly existing problems in multi-channel protocols.

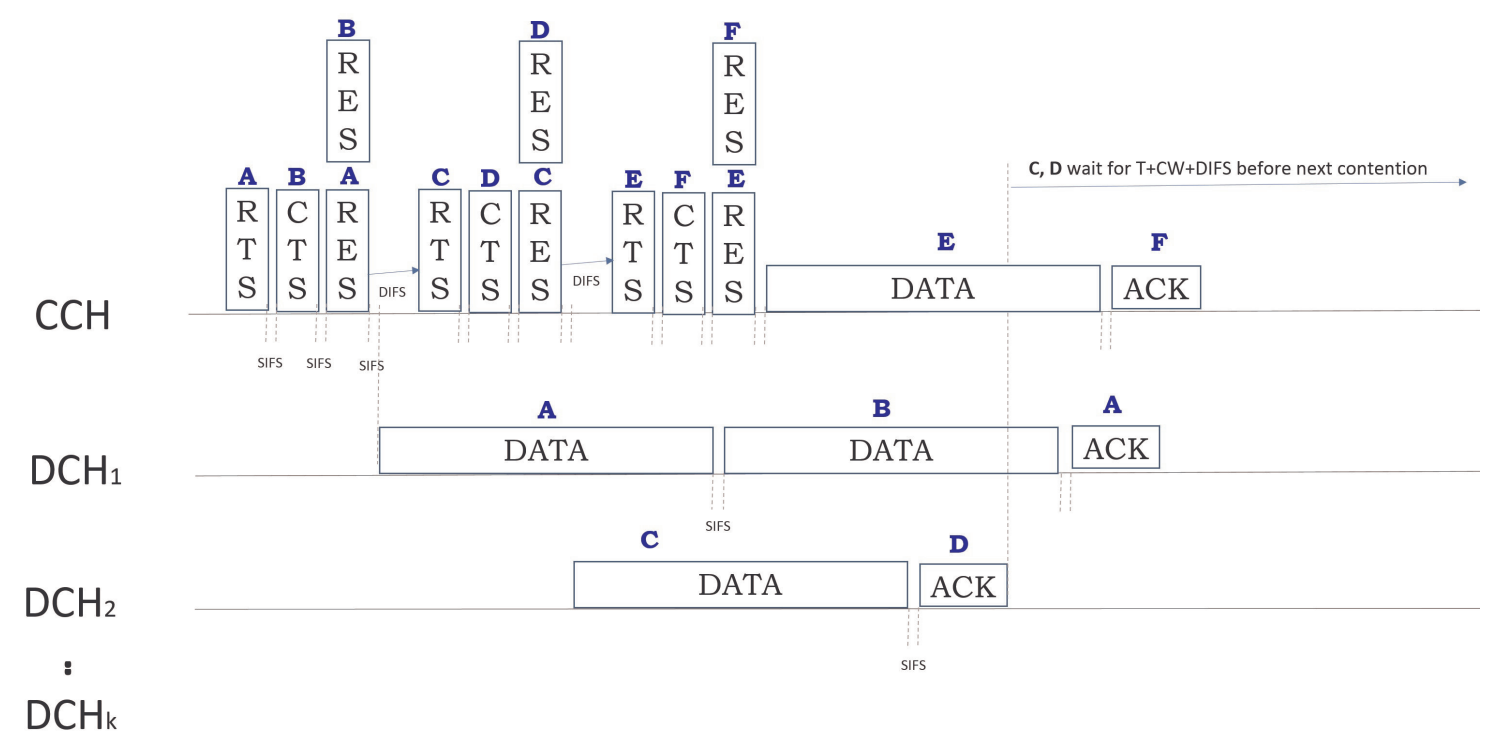

Figure 1. The spectrally efficient asynchronous multi-channel medium access control (SA-MMAC) protocol. CCH: control channel; RES: reservation; CTS: clear-to-send; RTS: request-to-send; ACK: acknowledgment; DIFS: Data Inter-Frame Space; SIFS: Short Inter-Frame Space. 


\subsection{Issue of Multi-Channel Hidden Terminal and Deafness Due to Use of a Single Radio}

The use of single radio results in multi-channel hidden terminal and deafness problems. At any given time, a half-duplex transceiver can only either send or receive on only one channel. A hidden terminal occurs in RTS/CTS as two nodes sending RTS at the same time cause a collision at the third node, or at least one node is deaf to the other RTS. It is also possible that when a node sends the CTS, a neighbor may send a RTS simultaneously. Also, nodes in data transfer are deaf to the exchanges on the control channel and may select a busy channel inadvertently in the next frame exchange. In our protocol, the nodes returning from data transfer wait a mandatory time for the maximum data frame on the control channel before contending again for transfer. By this approach, one node that has just completed data transfer (lets say node A) and is returning to the control channel, and another node which has obtained the data channel at the same time (lets say node Z) can get to know each other. Before node A starts another RTS, node $Z$ will be back to the control channel and will be ready to hear the control exchanges. This additional waiting time, while causing overhead, serves to eliminate the multi-channel hidden terminal and results in a highly collision-free operation.

\subsection{Issue of Increased Control Overhead}

In order to transfer data, a node has to incur control overhead due to the time spent in back-off, contention, and control signals such as RTS and CTS. For small data frames, more time is spent in sending the control signals. The collisions that happen during the contention lower the spectrum utilization. Nodes that obtain the channel often succeed and the failed nodes starve for a long time. Our protocol has two main overheads apart from contention and back-offs. Nodes returning from data transfer have to wait for one maximum data frame time. Nodes have to wait when control channel is used for data transfer. In order to offset the effect of these overheads, an impartiality is added to the protocol. Once a pair of nodes agrees on data transfer, the receiver can send an extra frame to the sender without the cost of contentions and back-offs. When a receiver needs to respond to an RTS, it checks if it has any data to the sender, adds it to data transfer time, and the CTS is sent. When the first data frame from sender (A) to receiver (B) is complete, receiver (B) sends the second frame (A) to the sender. The transfer of second frame from node $B$ is a piggybacked acknowledgment to node $A$. Node A knows that the transfer of first frame is successful. Node A then sends the ACK to B to confirm the receipt of second frame. In the absence of a data packet to sender (A), an ordinary ACK packet is sent from receiver (B). Thus, the second frame from node B effectively skips all the contention, waiting, back-off, and RTS-CTS signals. A visible downside of this approach is that other nodes suffer a delay in acquiring the channel due to transfer of two time frames in a single negotiation and the sender also needs to wait for an additional frame time before the next data exchange. This bi-directional approach was first proposed in [11] but using a dedicated control channel. The ill-effects of dedicated control channel have been previously discussed.

\subsection{Comparison of Features}

Our protocol uses an asynchronous mode of operation and employs a common agreed-upon channel for control signals. Nodes use distributed random access mechanism as in IEEE 802.11 for contention. This protocol supports broadcasts and does not need any temporal synchronization. However, a moderate channel switching delay is present. A comparison of features of the SA-MMAC protocol with the single-channel IEEE 802.11, the reliable channel-based multi-channel MAC (m-RCR), and the asynchronous multi-channel MAC (AMMAC) protocol is shown in Table 1. 
Table 1. Comparison of features of IEEE 802.11, asynchronous multi-channel MAC (AMMAC), and SA-MMAC. m-RCR: reliable channel-based multi-channel MAC.

\begin{tabular}{lcccc}
\hline Feature & IEEE 802.11 & AMMAC & m-RCR & SA-MMAC \\
\hline Issue of multi-channel hidden terminal & Not applicable & No & No & Very less \\
Control channel overhead & High & High & Less & High \\
More than one data frame in a handshake & No & No & No & Yes \\
Number of transceivers & 1 & 1 & 1 & 1 \\
Exclusive control channel needed & Not applicable & No & Yes & No \\
Channel selection * & Random & Random & Random & Last Used \\
\hline
\end{tabular}

* Subject to availability of common channels.

\section{Simulator Validation}

The SA-MMAC protocol follows IEEE 802.11 in its underlying back-off and contention mechanism with necessary modifications in control frames and handshakes for multi-channels. A custom-made packet-level simulator is done in python. We validate the simulator by comparing our IEEE 802.11 DCF and RTS/CTS implementation with some of existing seminal works [12-15]. The simulation parameters are set as in referred works. The results from our simulator in Figure 2a-c match with results of [12] (Figure 6), [13] (Figure 5), and [14] (Figure 6), respectively. Similarly, IEEE 802.11 saturation throughput, access delay, frame drop ratio, and Jain's fairness index (JFI) from the simulation (Figure 3a-d) accurately match with those of Akeel (refer to IEEE 802.11 graphs in Figure 3, Figure 4, and Figure 6 [15]). By establishing the accuracy and correctness of back-off and contention mechanism of IEEE 802.11, we establish the correctness of the simulator as the multi-channel protocols share the same IEEE 802.11 contention mechanism with necessary changes as per the demands of the protocol.

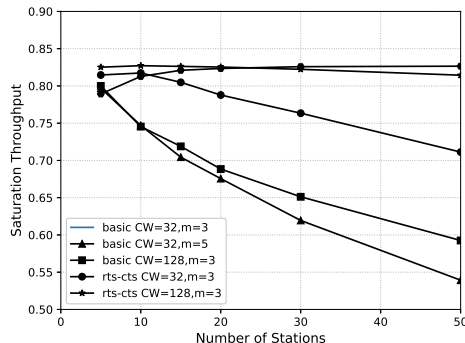

(a)

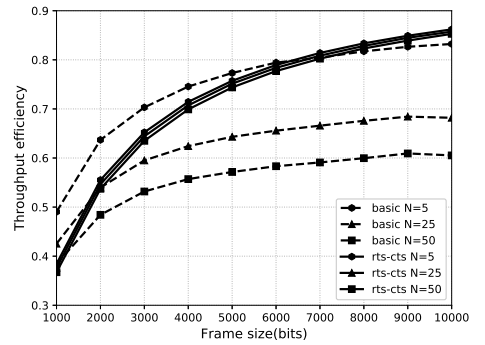

(b)

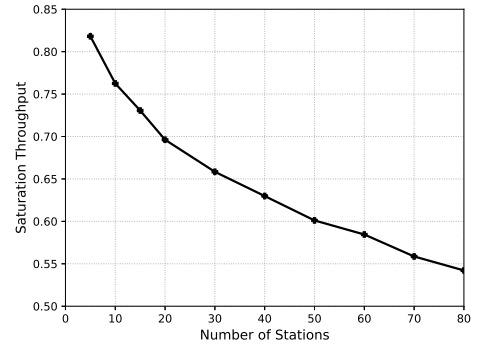

(c)

Figure 2. Comparison of simulation results with past works. (a) Comparison with Bianchi's work; (b) Comparison with Chatzimisios's work; (c) Comparison with Wu's work.

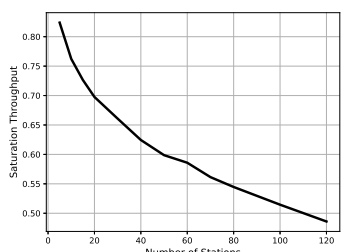

(a)

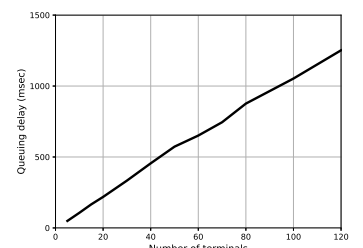

(b)

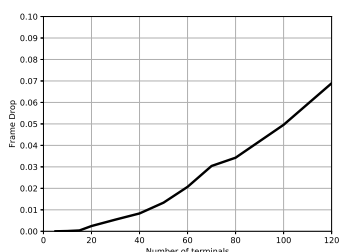

(c)

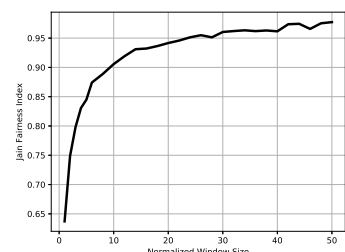

(d)

Figure 3. Comparison with Akeel's work. (a) Saturation Throughput; (b) Access Delay; (c) Frame Drop Ratio; (d) Jain's Fairness Index.

\section{Simulation Results and Discussion}

We detail the underlying assumptions for the performance evaluation. The simulation parameters are listed in Table 2.

- The nodes are in transmission range of each other in a single collision domain. 
- The channel assignment follows a per-packet basis.

- Saturated traffic conditions are assumed, so nodes will have a frame to transmit all the time.

- The frames exchanged are: RTS, CTS, ATS, DATA, and ACK. No other frames are considered.

- All nodes use the same PHY layer using the direct-sequence spread spectrum (DSSS) at a rate of 1 Mbps.

- Both data and control frames are sent at a rate of $1 \mathrm{Mbps}$. All nodes transmit with the same data rate. Varying data rates at various nodes at the same time are not considered. In case of collision, the frames are discarded. The capture effect is not considered.

- All simulations are run for a time of 10,000 data frames. The chosen frame count is validated and found to provide sufficient accuracy in populating the performance measures.

Table 2. Simulation parameters.

\begin{tabular}{ll}
\hline packet payload & 8224 \\
PHY header & 192 bits \\
MAC header & 224 bits \\
RTS & 168 bits + PHY header \\
CTS & 120 bits + PHY header \\
ATS & 120 bits + PHY header \\
ACK & 112 bits + PHY header \\
\hline Channel bit rate & $1 \mathrm{Mbit} / \mathrm{s}$ \\
Slot time & $20 \mu \mathrm{s}$ \\
SIFS & $10 \mu \mathrm{s}$ \\
DIFS & $50 \mu \mathrm{s}$ \\
\hline
\end{tabular}

We compare the SA-MMAC with the single-channel IEEE 802.11, and the following multi-channel asynchronous MAC protocols: (1) reliable channel-based multi-channel MAC (m-RCR) [9] and (2) asynchronous multi-channel MAC (AMMAC) [6]. The chosen protocols m-RCR and AMMAC belong to the same asynchronous multi-channel category using single half duplex transceiver and exhibit some characteristics of our proposed SA-MMAC. m-RCR reserves multiple steps per successful handshake and transmits RES again over selected intervals to avoid a multi-channel hidden terminal problem. It has one channel reserved for control exchanges and rest of the available channels for data transfer. In the simulations, we keep the $m$ factor of $m-R C R$ to 5 as suggested in [9]. The AMMAC reuses the control channel and the success node pair wait for a maximum data transfer time before starting contention. The m-RCR nor AMMAC protocols do not need time synchronization and use the IEEE 802.11 contention mechanism.

Figure 4a shows the normalized throughput for a fully connected network using three concurrent channels with increasing network load up to 80 nodes. When the network load is low, all protocols have a similar performance. As the network approaches saturation, SA-MMAC yields a significant performance over all the other protocols. The gain in performance over IEEE 802.11 is mainly due to usage of multiple channel transmissions. The normalized saturated throughput (0.7) is always capped below 1 . The $\mathrm{m}$-RCR protocol reserves one channel exclusively for control exchanges, which means one channel less for data transfer. The performance gain of SA-MMAC over AMMAC is due to the fact that the nodes are involved in full duplex transmissions on data channels skipping the control channel negotiations and back-off overhead. Figure $4 b, c$ shows the results for the same network scenario when using 4 and 12 channels. As we can observe, for high node density of 80 nodes using 12 channels, the saturation throughput of SA-MMAC (7.3740) has about $1245 \%$ more throughput than 802.11 (0.5479), 94\% more than m-RCR(3.7908), and 18\% more than AMMAC (6.2430). 


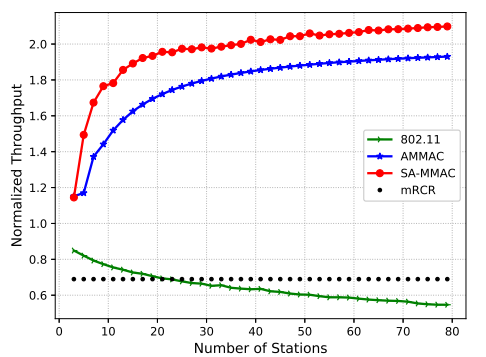

(a)

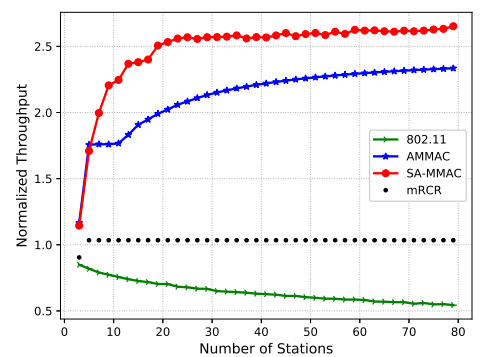

(b)

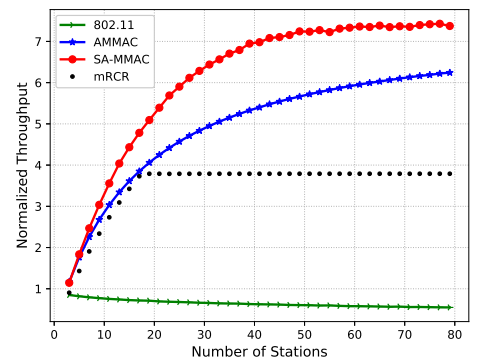

(c)

Figure 4. Comparison of the saturated throughput for SA-MMAC with others. (a) 3 channels; (b) 4 channels; (c) 12 channels.

Figure 5a shows the comparison of channel access delay for 3 channels as the node density increases from 5 to 80 . As the traffic load increases, the access delay for IEEE 802.11 sharply rises as the collisions happen during channel negotiation and data transfer. All the nodes have to wait until the complete data transfer time as indicated in the RTS or CTS message and then start a contention. In multi-channels, m-RCR has the worst access delay as the nodes that succeed contention occupy a whopping $\mathrm{m}$ times data transfer on the data channel. Nodes that lose the contention have to wait for the time of $\mathrm{m}$ data frames, not to mention the inherent weakness in contention mechanism of the IEEE 802.11(briefly discussed later). SA-MMAC has similar channel access delay to AMMAC but is marginally better. The similarity is due to the fact SA-MMAC employs a mandatory maximum time frame wait after successful data transfer, similar to that of AMMAC. The waiting period for the control channel in SA-MMAC is reduced to about half of that of AMMAC. Moreover, we need to consider the fact that the data transfer is bi-directional in data channels. Despite a full duplex mode operation in data channels and control channel usage, SA-MMAC still performs comparably to AMMAC. We repeat the experiments for 4 and 12 channels as in Figure $5 b, c$ and a similar trend is observed. The proposed protocol thus scales well to 12 channels with the same kind of performance.

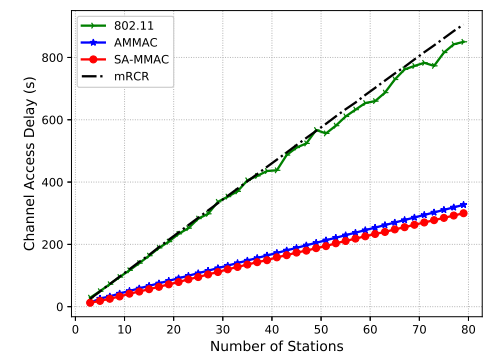

(a)

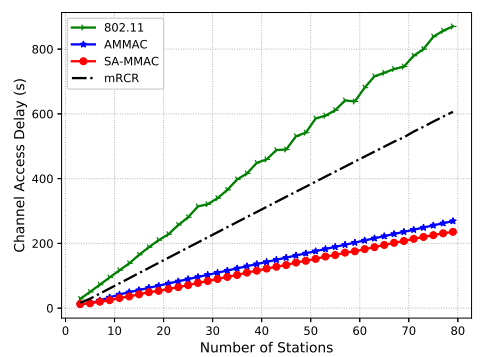

(b)

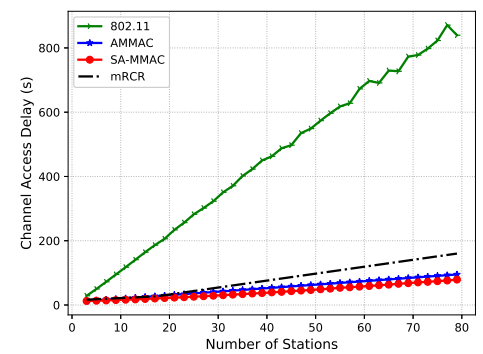

(c)

Figure 5. Comparison of channel access delay for SA-MMAC with other protocols. (a) 3 channels; (b) 4 channels; (c) 12 channels.

Figure 6a shows Jain's fairness index (JFI) versus the number of nodes in the network. As the node traffic increases, the fairness index in IEEE 802.11 drastically reduces due to its poor contention mechanism. The phenomenon of "rich get richer and the poor get poorer" occurs. Nodes that successfully transfer data get an early chance to negotiate as the CW is set to a minimum, whereas the failed nodes have to wait longer and any further collision worsens its chance of getting the channel access. m-RCR is seen to have a better JFI than others. JFI is calculated based on the transmitted packets in a time window and any dropped packets will be excluded. The fact is that m-RCR drops more packets and as a side effect, there is an increase in JFI. This is explained later. AMMAC increases the JFI by a modified contention mechanism. The nodes successful in data transfer must wait the maximum data frame time before they start their next contention. This facilitates the other nodes 
getting the transmission channel. AMMAC has a better JFI than SA-MMAC (about 25\% better) due to the fact there is only one data frame transmission per handshake in AMMAC whereas in SA-MMAC, the receiver can use the same handshake and send an extra data frame, skipping the contention overhead. The reduction of JFI in SA-MMAC is because only the receiver node in successful negotiation gets preferential treatment, skipping the overhead. A similar trend is observed for 4 and 12 multiple channels in Figure 6a,b.

The adaptability of SA-MMAC for a large density of nodes and high traffic conditions can be observed from the results in Tables 3 and 4. Table 3 shows the frame drop ratio (FDR) for a network of 100 nodes under three scenarios (3, 4, and 12 channels). IEEE 802.11 has frame drop ratio of $(5.31 \%)$ irrespective of the number of channels, as it uses only one channel and the remainder of the bandwidth remains unused. All multi-channel protocols have a nil frame drop ratio for 100 nodes.

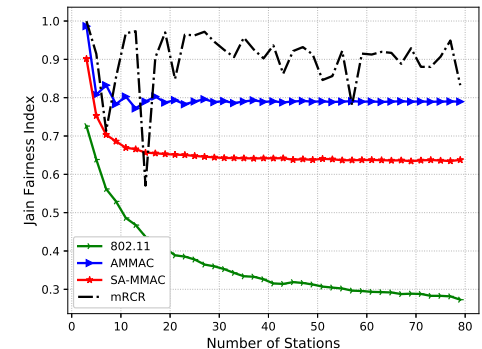

(a)

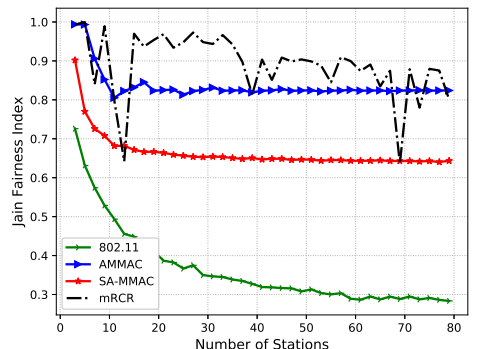

(b)

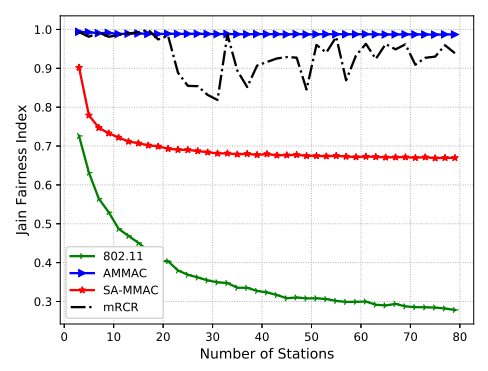

(c)

Figure 6. Comparison of Jain's fairness index for SA-MMAC with other protocols. (a) 3 channels; (b) 4 channels; (c) 12 channels.

Table 3. Comparison of the frame drop ratio (FDR) for 100 nodes, and 4 channels.

\begin{tabular}{cccccc}
\hline Nodes & Channels & Basic DCF & AMMAC & m-RCR & SA-MMAC \\
\hline 100 & 3 & 5.31 & 0 & 0 & 0 \\
100 & 4 & 5.31 & 0 & 0 & 0 \\
100 & 12 & 5.31 & 0 & 0 & 0 \\
\hline
\end{tabular}

Table 4. Comparison of the FDR for 500 nodes, and 4 channels.

\begin{tabular}{cccc}
\hline Basic DCF & AMMAC & m-RCR & SA-MMAC \\
\hline 57.25 & 0.31 & 0.64 & 0.17 \\
\hline
\end{tabular}

In a densely populated network of 500 nodes, IEEE 802.11 fares poorly, dropping about $57 \%$ of the packets. In m-RCR, packets wait longer and longer due to extended transmissions on the data channels; the re-transmission attempts fail and the packets get dropped rampantly. Hence, m-RCR drops a whopping $64 \%$ of the packets. The SA-MMAC outperforms its counterpart AMMAC with a $45 \%$ lower frame drop ratio (FDR). By virtue of its watch-and-proceed contention mechanism and neighbors' better awareness of control exchanges, SA-MMAC results in almost a collision-free operation.

Figure 7 shows the performance of SA-MMAC for four channels for a network of 100 nodes with a varying number of channels. As we can observe, with respect to the performance of a network with the use of multi-channels, as parallel data transmissions occur, the availability of more channels translates to an increase in saturation throughput and a decrease in channel access delay. The fairness index almost remains the same. Table 5 shows the performance of SA-MMAC for the same scenario of 100 nodes and four channels with different packet payload sizes. We can notice that the trend is similar irrespective of the frame sizes. 


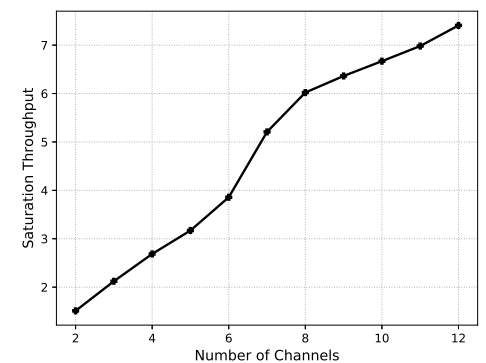

(a)

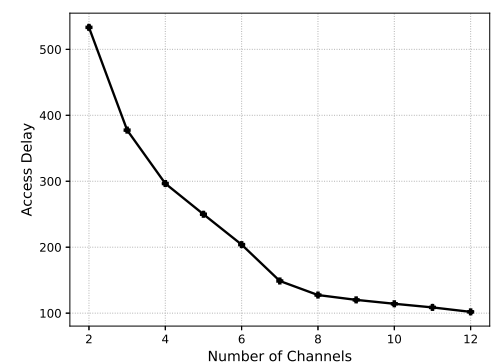

(b)

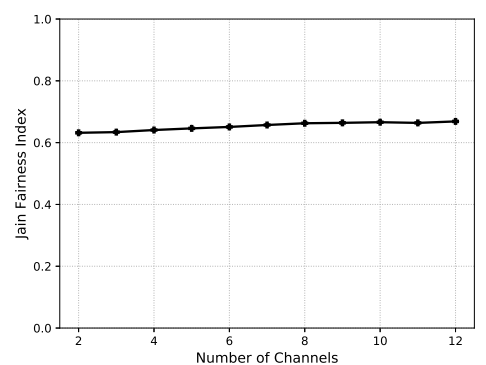

(c)

Figure 7. Performance of SA-MMAC for 100 nodes. (a) Saturation throughput; (b) access delay; (c) frame drop ratio.

Table 5. Performance of SA-MMAC at different packet payload sizes.

\begin{tabular}{cccc}
\hline Frame Size (bits) & Saturation Throughput & Access Delay (s) & JFI \\
\hline 2000 & 2.6615 & 299.32 & 0.64 \\
3000 & 2.6607 & 299.42 & 0.64 \\
4000 & 2.6668 & 298.72 & 0.64 \\
5000 & 2.6704 & 298.32 & 0.64 \\
6000 & 2.6611 & 299.42 & 0.64 \\
7000 & 2.6614 & 299.37 & 0.64 \\
8000 & 2.6676 & 298.62 & 0.64 \\
\hline
\end{tabular}

We considered another scenario of a populated network of 200 nodes with different number of available channels and compared the protocols. As we can observe in Figure $8 \mathrm{a}$, the increase in the number of channels does no good to the IEEE 802.11. As the network is heavily loaded, the performance of IEEE 802.11 sharply drops with normalized saturation throughput reduced to 0.4 and access delay increased by more than $1750 \mathrm{~s}$. The m-RCR throughput increases with the increase in number of channels, and does not go beyond half of the spectrum utilization, with a saturation throughput of less than 4 for 8 channels. For AMMAC, the performance is saturated at 10 channels and cannot take advantage of more channels thereafter. This is due to the mandatory waiting time for the nodes returning from data transfer and moreover, the high traffic leads to increase in RTS collisions, more retransmission attempts, and a drop in packets. The saturation throughput of SA-MMAC can be seen as increasing even beyond 10 channels, which confirms its suitability for a higher number of channels without the control channel becoming a bottle-neck. We consider the channel access delay of protocols with varying numbers of channels. This is given in Figure $8 \mathrm{~b}$. The delay characteristics of IEEE 802.11 do not change due to the reasons discussed earlier. The access delay of AMMAC (and also $\mathrm{m}-\mathrm{RCR}$ to an extent) converges with SA-MMAC at a higher number of channels. However, we need to consider the fact that AMMAC throughput is already saturated and packets are dropped heavily. As for $\mathrm{m}-\mathrm{RCR}$, the packet drop is even worse for higher density networks. Thus we can establish that SA-MMAC transmits more packets than other protocols with much less of an access delay. 


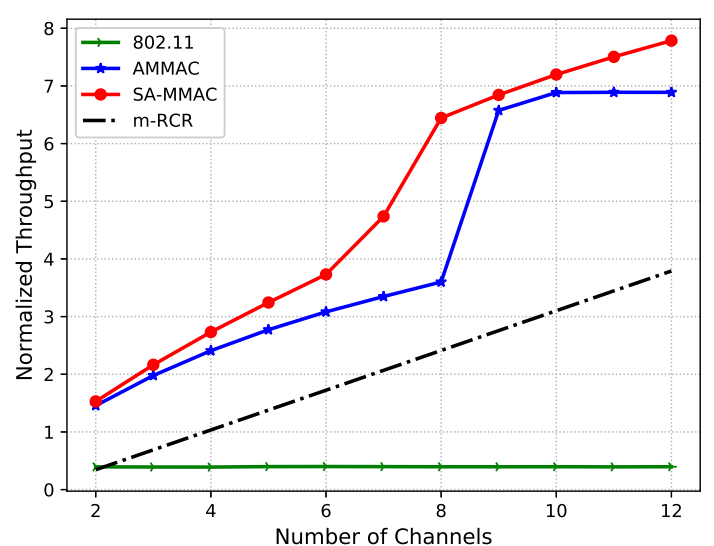

(a)

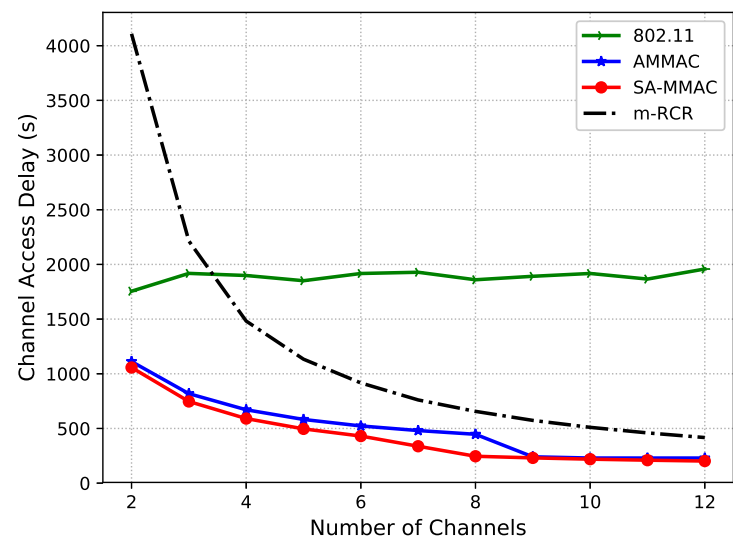

(b)

Figure 8. Performance of protocols for a densely populated network of 200 nodes. (a) Saturation Throughput; (b) Access Delay.

\section{Conclusions and Future Work}

In this work, we proposed a full duplex, asynchronous multi-channel MAC for wireless networks, making use of a single antenna. The protocol increases the spectral efficiency by low overhead signaling and re-use of spatial channels. The new protocol extends the IEEE 802.11 contention mechanism and performs load balancing by assigning multiple transmissions over non-overlapping channels without any explicit co-ordination. The IEEE 802.11 back-off algorithm is modified to eliminate the multi-channel hidden terminal problem and potential interference from neighbors during the handshake. The use of the half-duplex transceiver reduces the cost and size of the node. The energy conservation of the node and its low cost is especially significant for mass deployment and in remote environments which mostly lack power infrastructures. The simulator correctness is established by comparison of results with some past seminal works. The results suggest that the proposed protocol achieves a gain of $1245 \%$ in terms of saturation throughput and about $18 \%$ to $95 \%$ better gains compared to its counterparts AMMAC and m-RCR, respectively. It entails highly collision-free operation. The high performance of this protocol under loaded conditions makes it a suitable candidate for dense wireless networks. The mobility of the nodes and multi-hop scenarios are not considered. The inherent weakness of the IEEE 802.11 contention mechanism is still a big factor in reduced spectral efficiency. A better back-off algorithm or a self-adaptive contention window would further enhance the performance of the protocol, which we will leave for future study.

Acknowledgments: The authors would like to thank King Fahd University of Petroleum and Minerals, Dhahran, Saudi Arabia, for their support.

Author Contributions: Abdullah Devendiran conceived the idea, implemented the protocols, and made the write-up for paper. Tarek Sheltami mentored and supervised the work completely, and reviewed the paper. Ashraf Mahmoud provided a similar simulation tool and reviewed the paper.

Conflicts of Interest: The authors declare no conflict of interest.

\section{References}

1. Institute of Electrical and Electronics Engineers (IEEE). IEEE Standard 802.11: Wireless LAN Medium Access Control (MAC) and Physical Layer (PHY) Specifications; IEEE: New York, NY, USA, 1999.

2. Almotairi, K.H.; Shen, X.S. A distributed multi-channel MAC protocol for ad hoc wireless networks. IEEE Trans. Mob. Comput. 2015, 14, 1-13.

3. Murdiyat, P.; Chung, K.S.; Chan, K.S. A multi-channel MAC for multi-hop wireless sensor networks minimizing hidden node collision. In Proceedings of the IEEE 2016 22nd Asia-Pacific Conference on Communications (APCC), Yogyakarta, Indonesia, 25-27 August 2016; pp. 535-540. 
4. Dang, D.N.M.; Hong, C.S. H-MMAC: A hybrid multi-channel MAC protocol for wireless ad hoc networks. In Proceedings of the 2012 IEEE International Conference on Communications (ICC), Ottawa, ON, Canada, 10-15 June 2012; pp. 6489-6493.

5. Arafeh, A.; Oughali, F.; Sheltami, T.; Mahmoud, A.; Daabaj, K. A contention free multi-channel MAC protocol with improved negotiation efficiency for wireless ad-hoc networks. In Proceedings of the International ACM Conference on Ambient Systems, Networks and Technologies (ACM ANT 2010), Paris, France, 8-10 November 2010.

6. Nguyen, D.; Garcia-Luna-Aceves, J.; Obraczka, K. Collision-free asynchronous multi-channel access in ad hoc networks. In Proceedings of the IEEE Global Telecommunications Conference, GLOBECOM 2009, Honolulu, HI, USA, 30 November-4 December 2009; pp. 1-6.

7. Wormsbecker, I.; Williamson, C. On channel selection strategies for multi-channel MAC protocols in wireless ad hoc networks. In Proceedings of the IEEE International Conference on Wireless and Mobile Computing, Networking and Communications (WiMob'2006), Montreal, QC, Canada, 19-21 June 2006; IEEE: Piscataway, NJ, USA, 2006.

8. Luo, T.; Motani, M.; Srinivasan, V. Cooperative asynchronous multichannel MAC: Design, analysis, and implementation. IEEE Trans. Mob. Comput. 2009, 8, 338-352.

9. Yang, B.; Li, B.; Yan, Z.; Yang, M.; Zuo, X. A reliable channel reservation based multi-channel MAC protocol with a single transceiver. In Proceedings of the IEEE 2015 11th International Conference on Heterogeneous Networking for Quality, Reliability, Security and Robustness (QSHINE), Taipei, Taiwan, 19-20 August 2015; pp. 265-271.

10. Zhang, Y.; Lazos, L.; Chen, K.; Hu, B.; Shivaramaiah, S. Multi-Channel Medium Access without Control Channels: A Full Duplex MAC Design. IEEE Trans. Mob. Comput. 2017, 16, 1032-1046.

11. Kuang, T.; Williamson, C. A bidirectional multi-channel MAC protocol for improving TCP performance on multihop wireless ad hoc networks. In Proceedings of the 7th ACM International Symposium on Modeling, Analysis and Simulation of Wireless and Mobile Systems, Venice, Italy, 4-6 October 2004; ACM: New York, NY, USA, 2004; pp. 301-310.

12. Bianchi, G. Performance analysis of the IEEE 802.11 distributed coordination function. IEEE J. Sel. Areas Commun. 2000, 18, 535-547.

13. Chatzimisios, P.; Vitsas, V.; Boucouvalas, A.C. Throughput and delay analysis of IEEE 802.11 protocol. In Proceedings of the 2002 IEEE 5th International Workshop on Networked Appliances, Liverpool, UK, 30-31 October 2002; pp. 168-174.

14. Wu, H.; Cheng, S.; Peng, Y.; Long, K.; Ma, J. IEEE 802.11 distributed coordination function (DCF): Analysis and enhancement. In Proceedings of the ICC 2002, IEEE International Conference on Communications, New York, NY, USA, 28 April-2 May 2002; Volume 1, pp. 605-609.

15. Al-Akeel, A. Optimizing Backoff Procedure for Enhanced Throughput and Fairness in Wireless LANs. Master's Thesis, King Fahd University of Petroleum \& Minerals, Dhahran, Saudi Arabia, 2007.

(C) 2018 by the authors. Licensee MDPI, Basel, Switzerland. This article is an open access article distributed under the terms and conditions of the Creative Commons Attribution (CC BY) license (http://creativecommons.org/licenses/by/4.0/). 